\title{
On velocity space interrogation regions of fast-ion collective Thomson scattering at ITER
}

Salewski, Mirko; Nielsen, Stefan Kragh; Bindslev, Henrik; Furtula, Vedran; Gorelenkov, N.N.; Korsholm, Søren Bang; Leipold, Frank; Meo, Fernando; Michelsen, Poul; Moseev, Dmitry

Total number of authors:

11

Published in:

Nuclear Fusion

Link to article, DOI:

$10.1088 / 0029-5515 / 51 / 8 / 083014$

Publication date:

2011

Link back to DTU Orbit

Citation (APA):

Salewski, M., Nielsen, S. K., Bindslev, H., Furtula, V., Gorelenkov, N. N., Korsholm, S. B., Leipold, F., Meo, F., Michelsen, P., Moseev, D., \& Stejner Pedersen, M. (2011). On velocity space interrogation regions of fast-ion collective Thomson scattering at ITER. Nuclear Fusion, 51(8), 083014. https://doi.org/10.1088/0029$5515 / 51 / 8 / 083014$

\section{General rights}

Copyright and moral rights for the publications made accessible in the public portal are retained by the authors and/or other copyright owners and it is a condition of accessing publications that users recognise and abide by the legal requirements associated with these rights.

- Users may download and print one copy of any publication from the public portal for the purpose of private study or research.

- You may not further distribute the material or use it for any profit-making activity or commercial gain

- You may freely distribute the URL identifying the publication in the public portal 
9 June 2011

\title{
On velocity space interrogation regions of fast-ion collective Thomson scattering at ITER
}

\author{
M Salewski $^{1}$, S K Nielsen ${ }^{1}$, H Bindslev ${ }^{1}$, V Furtula ${ }^{1}$, \\ N N Gorelenkov ${ }^{2}$, S B Korsholm ${ }^{1}$, F Leipold ${ }^{1}$, F Meo ${ }^{1}$, \\ P K Michelsen ${ }^{1}$, D Moseev ${ }^{1}$, M Stejner ${ }^{1}$ \\ ${ }^{1}$ Association Euratom - Ris $\varnothing$ National Laboratory for Sustainable Energy, Technical \\ University of Denmark, DK-4000 Roskilde, Denmark \\ 2 Princeton Plasma Physics Laboratory, Princeton, NJ 08543-045, USA \\ E-mail: msal@risoe.dtu.dk
}

\begin{abstract}
The collective Thomson scattering (CTS) diagnostic proposed for ITER is designed to measure projected 1D fast-ion velocity distribution functions at several spatial locations simultaneously. The frequency shift of scattered radiation and the scattering geometry place fast ions that caused the collective scattering in well-defined regions in velocity space, here dubbed interrogation regions. Since the CTS instrument measures entire spectra of scattered radiation, many different interrogation regions are probed simultaneously. We here give analytic expressions for weight functions describing the interrogation regions, and we show typical interrogation regions of the proposed ITER CTS system. The backscattering system with receivers on the low field side is sensitive to fast ions with pitch $|p|=\left|v_{\|} / v\right|<0.5-0.9$ depending on the ion energy and the frequency shift of the scattered radiation. A forward scattering system with receivers on the high field side would be sensitive to co- and counter-passing fast ions in narrow interrogation regions with pitch $|p|>0.6-0.8$. Additionally, we use weight functions to reconstruct 2D fast-ion distribution functions, given two projected $1 \mathrm{D}$ velocity distribution functions from simulated simultaneous measurements with the back- and forward scattering systems.
\end{abstract}

PACS numbers: 52.25.Os, 52.40.Db, 52.50.Gj, 52.65.Cc, 52.70.Gw 


\section{Introduction}

In burning plasmas in ITER, fast ions from fusion reactions, from neutral beam injection (NBI) and from ion cyclotron resonance heating (ICRH) will provide more than $2 / 3$ of the total heating by transferring most of their energy to the bulk plasma. However, many aspects of fast-ion behaviour, which still pose challenges to theory, may lead to enhanced energy and fast-ion losses. For example, fast ions can develop energetic particle modes $[1,2]$ and can interact with Alfvén eigenmodes [3-7], with sawteeth instabilities [8-11], with kinetic ballooning modes $[4,12,13]$, with neoclassical tearing modes $[4,14]$ or with turbulent fluctuations [15-17]. The extrapolation of such phenomena to ITER plasmas is even more uncertain, and measurements of fast-ion velocity distributions in ITER are therefore essential $[18,19]$. Millimetre-wave collective Thomson scattering (CTS) has been demonstrated to provide such measurements at JET [20], TEXTOR [10,11,21-23] and ASDEX Upgrade [24-26].

The proposed ITER CTS system comprises a forward scattering system with receivers on the high field side and a backscattering system with receivers on the low field side [27-32]. Each system is designed to measure fast-ion velocity distributions in $7-10$ measurement volumes distributed along the minor radius $a$ on both the high field side and the low field side simultaneously, satisfying the ITER measurement requirements on resolution for fusion alpha diagnostic (time: $100 \mathrm{~ms}$, space: a/10) [33]. The CTS backscattering system is an enabled ITER diagnostic, and its in-port components are part of the updated ITER baseline design [34]. The forward scattering system [29,32], however, is not enabled. CTS measurements are sensitive to the fast-ion velocity distribution function projected onto the wave vector $\mathbf{k}^{\delta}=\mathbf{k}^{s}-\mathbf{k}^{i}$ where $s$ and $i$ refer to scattered and incident radiation, respectively. A frequency shift $\nu^{\delta}$ of scattered radiation can be related to an ion velocity $\mathbf{v}^{p}$ projected onto $\mathbf{k}^{\delta}: \nu^{\delta}=\nu^{s}-\nu^{i} \approx \mathbf{v}^{p} \cdot \mathbf{k}^{\delta} / 2 \pi$. The detected frequency shift and the projection angle between $\mathbf{k}^{\delta}$ and the magnetic field $\mathbf{B}$, $\phi=\angle\left(\mathbf{k}^{\delta}, \mathbf{B}\right)$, place the ions that caused the collective scattering in a well-defined region in velocity space, here dubbed interrogation region. Typical interrogation regions of the back- and forward scattering ITER CTS systems are presented using weight functions such as those used for fast-ion $\mathrm{D} \alpha$ (FIDA) diagnostic [35-38]. Weight functions are a convenient and illustrative way to relate the fast-ion distribution function and the geometry of the experiment to the measured CTS signal.

To develop analytic expressions for CTS weight functions in Sec. 4, we define relevant coordinate systems in Sec. 2 and illustrate the projection of velocity distribution functions onto $\mathbf{k}^{\delta}$ in Sec. 3. The analytic expressions derived here should also be useful for FIDA because CTS weight functions are identical with the geometric (Doppler shift) part of FIDA weight functions [38]. In Sec. 5 we show, using weight functions, that the ITER CTS backscattering system is sensitive to dynamics of fast ions with pitch $|p|=\left|v_{\|} / v\right|<0.5-0.9$, depending on the ion energy and the frequency shift of the scattered radiation. Here $v_{\|}$is the velocity component parallel to $\mathbf{B}$ and $v$ is the velocity magnitude. The proposed forward scattering system would reveal dynamics of co- and 
counter-passing fast ions in narrow interrogation regions with pitch $|p|>0.6-0.8$ as opposed to the backscattering system where the interrogation regions cover broad ranges of pitch angle and energy. CTS signals at ITER are dominated by alpha particles [30,31]. For a central alpha distribution function computed with TRANSP [39], most of the CTS signal at various frequency shifts originates from the low energy end of the interrogation regions which are thus effectively probed (Sec. 5). Simultaneous measurements with both systems would even make it possible to study anisotropy in the fast-ion velocity distribution and to reconstruct 2D velocity distributions (Sec. 6). We conclude in Sec. 7.

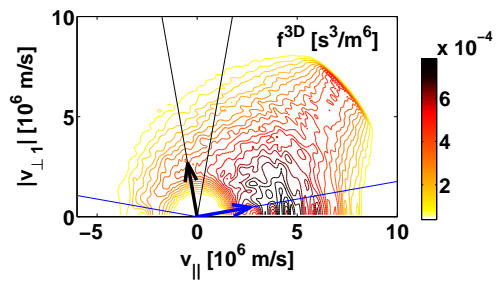

(a) $f^{3 D}\left(v_{\|}, v_{\perp 1}, v_{\perp 2}\right)$

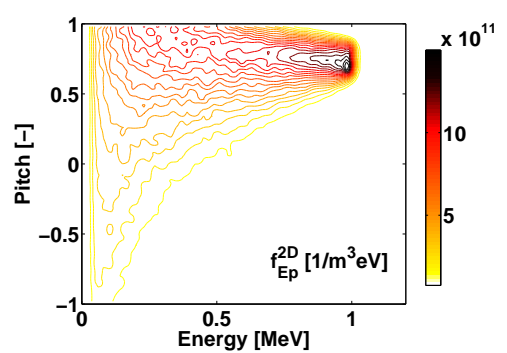

(c) $f_{E p}^{2 D}(E, p)$

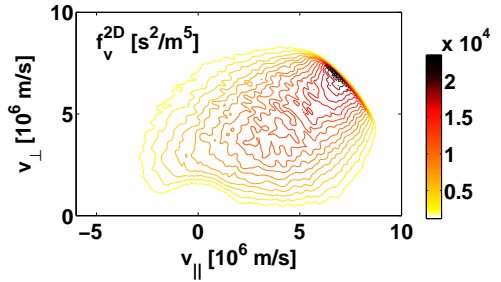

(b) $f_{v}^{2 D}\left(v_{\|}, v_{\perp}\right)$

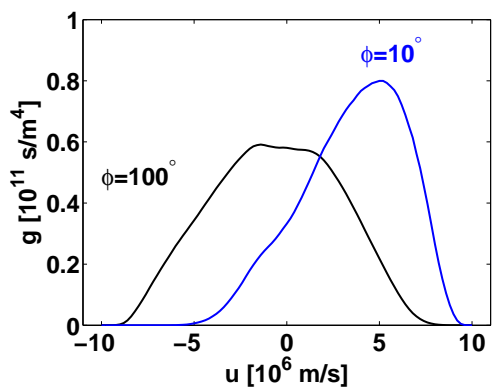

(d) $g(u)$

Figure 1. An ITER NBI ion distribution function at the minor radius coordinate $r / a=0.25$ in the midplane on the high field side computed with TRANSP shown in four coordinate systems. (a) Slice through the 3D, rotation invariant velocity distribution function $f^{3 D}\left(v_{\|}, v_{\perp 1}, v_{\perp 2}\right)$. The projection directions are indicated by the vector $\mathbf{k}^{\delta}$ and thin straight lines for $\phi=100^{\circ}$ (black) and $\phi=10^{\circ}$ (blue). Positive $u$-coordinates are on the branches containing the arrows and negative $u$-coordinates lie on the mirror-reflected branches. (b) $2 \mathrm{D}$ velocity distribution function $f_{v}^{2 D}\left(v_{\|}, v_{\perp}\right)$. (c) $2 \mathrm{D}$ velocity distribution function $f_{E p}^{2 D}(E, p)$. (d) $1 \mathrm{D}$ projected velocity distribution functions $g(u)$ corresponding to the projection directions indicated in (a).

\section{Coordinate systems}

In CTS experiments 1D projections $g(u)$ of full 3D fast-ion velocity distribution functions $f^{3 D}\left(v_{\|}, v_{\perp 1}, v_{\perp 2}\right)$ along $\mathbf{k}^{\delta}$ are estimated by measuring the spectral power density of scattered radiation. The Cartesian velocity component $v_{\|}$is parallel to $\mathbf{B}$ and $v_{\perp 1}$ and $v_{\perp 2}$ are perpendicular to $\mathbf{B}$, and $u$ is the $1 \mathrm{D}$ velocity component in the direction of $\mathbf{k}^{\delta}$. 
The projection is given by

$$
g(u)=\int f^{3 D}(\mathbf{v}) \delta\left(\frac{\mathbf{v} \cdot \mathbf{k}^{\delta}}{k^{\delta}}-u\right) d \mathbf{v},
$$

where $\mathbf{v}$ is the velocity and $\delta()$ is the Dirac delta function. The $u$-coordinate determines the frequency shift of scattered radiation in CTS experiments:

$$
\nu^{\delta} \approx u k^{\delta} / 2 \pi \text {. }
$$

Figure 1 shows an NBI ion distribution function in four different representations. The distribution function has been computed with TRANSP simulating an ITER plasma during the injection. Figure $1(\mathrm{a})$ is a $2 \mathrm{D}$ slice through the $3 \mathrm{D}$ distribution function $f^{3 D}\left(v_{\|}, v_{\perp 1}, v_{\perp 2}\right)$. As we assume $f^{3 D}$ to be rotationally symmetric about $\mathbf{B}$, we are free to choose $v_{\perp 1}$ to lie in the plane spanned by $\mathbf{k}^{\delta}$ and $\mathbf{B}$. For the same reason we can choose $\left|v_{\perp 1}\right|$ as coordinate axis since the half plane with negative $v_{\perp 1}$ is a mirror reflection of that with positve $v_{\perp 1}$. Two typical projection directions for a forward- and a backscattering ITER CTS system are indicated by vector arrows and two branches of thin straight lines for each projection angle in Fig. 1(a). The corresponding 1D distribution functions $g$ are shown in Fig. $1(\mathrm{~d})$. As the relation between $f^{3 D}$ and $g$ is by no means obvious, we will elaborate on the mapping from $\left(v_{\|}, v_{\perp 1}, v_{\perp 2}\right)$-coordinates of $f^{3 D}$ onto the $u$-coordinate of $g$ in the following section.

As we assume the fast-ion distribution function to be rotationally invariant about $\mathbf{B}$, it is sufficient and convenient to work with the fast-ion distribution functions $f_{v}^{2 D}\left(v_{\|}, v_{\perp}\right)$ and $f_{E p}^{2 D}(E, p)$ shown in Fig. $1(\mathrm{~b})$ and (c). The perpendicular velocity components $v_{\perp 1}$ and $v_{\perp 2}$ are related to $v_{\perp}$ through the gyroangle $\gamma$ by $v_{\perp 1}=v_{\perp} \cos \gamma$ and $v_{\perp 2}=v_{\perp} \sin \gamma$, so $v_{\perp}^{2}=v_{\perp 1}^{2}+v_{\perp 2}^{2}$. The energy is $E=\frac{1}{2} m v^{2}$, and the pitch is $p=\frac{v_{\|}}{v}$ with $v^{2}=v_{\|}^{2}+v_{\perp}^{2}$. The $(E, p)$ - and $\left(v_{\|}, v_{\perp}\right)$-coordinate systems are truly $2 \mathrm{D}$ with no implied third direction. We derive weight functions describing the interrogation regions in these coordinate systems (Sec. 5). The representations of the fast-ion distribution function in Figs. 1(a)(c) are equivalent. One can obtain the velocity distribution function $f_{v}^{2 D}$ from $f^{3 D}$ by transforming to cylindrical coordinates and integrating over the by assumption ignorable gyroangle $\gamma, f_{v}^{2 D}=2 \pi v_{\perp} f^{3 D}$, and the velocity distribution function $f_{E p}^{2 D}$ is related to $f_{v}^{2 D}$ through a $2 \mathrm{D}$ coordinate transformation. The respective Jacobians are included in the definitions $f_{v}^{2 D}$ and $f_{E p}^{2 D}$, so that the ion particle density is respectively given by

$$
\begin{aligned}
n & =\int f^{3 D}\left(v_{\|}, v_{\perp 1}, v_{\perp 2}\right) d v_{\perp 1} d v_{\perp 2} d v_{\|}=\int f_{v}^{2 D}\left(v_{\|}, v_{\perp}\right) d v_{\perp} d v_{\|} \\
& =\int f_{E p}^{2 D}(E, p) d E d p=\int g(u) d u .
\end{aligned}
$$

\section{Mapping from $\left(v_{\|}, v_{\perp 1}, v_{\perp 2}\right)$-coordinates and $\left(v_{\|}, v_{\perp}\right)$-coordinates onto $u$-coordinates}

We illustrate the projection trace of a gyrating ion in $\left(v_{\|}, v_{\perp 1}, v_{\perp 2}\right)$-space onto $u$-space along $\mathbf{k}^{\delta}$ in Fig. 2. The gyroorbit about the $v_{\|}$-axis can be parametrized by $\left(v_{\|}^{p}, v_{\perp}^{p}, \gamma^{p}\right)$ 


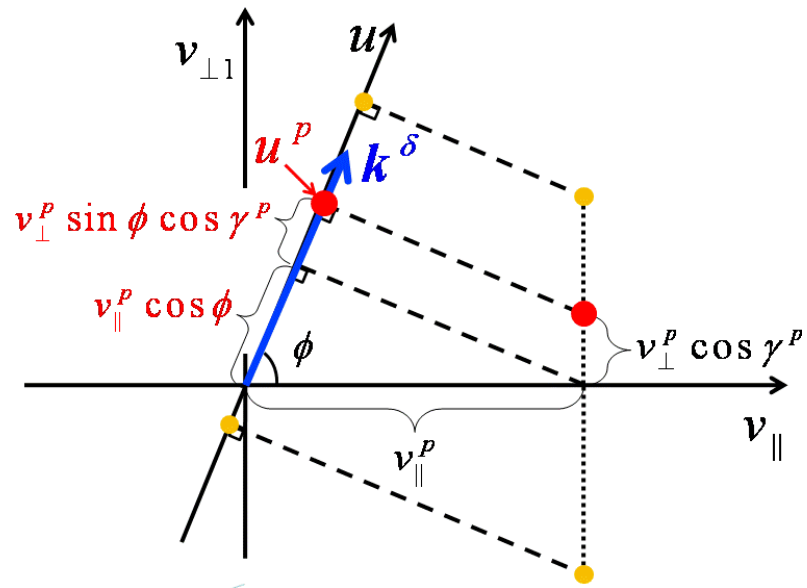

(a)

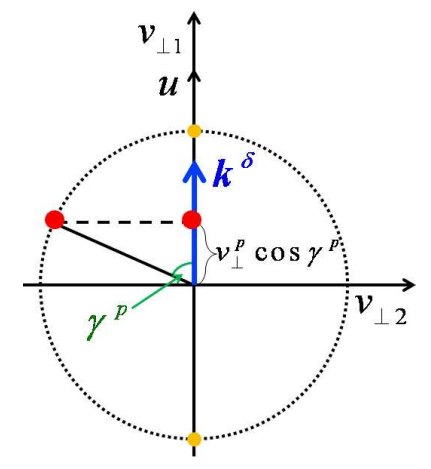

(b)

Figure 2. Illustration of the mapping from $\left(v_{\|}, v_{\perp 1}, v_{\perp 2}\right)$-space onto $u$-space along $\mathbf{k}^{\delta}$ (blue). (a) $\left(v_{\|}, v_{\perp 1}\right)$-plane: The point $\left(v_{\|}^{p}, v_{\perp}^{p} \cos \gamma^{p}\right)$ (large red dot), which is always on the dotted line between the extremal points $\left(v_{\|}^{p}, v_{\perp}^{p}\right)$ and $\left(v_{\|}^{p},-v_{\perp}^{p}\right)$ (small orange dots), is projected onto the $u$-coordinate at angle $\phi$ to the $v_{\|}$-axis. The dashed lines are perpendicular to the $u$-axis. As $\cos \gamma^{p} \in[-1,1], u^{p} \in\left[v_{\|}^{p} \cos \phi-\right.$ $\left.v_{\perp}^{p} \sin \phi, v_{\|}^{p} \cos \phi+v_{\perp}^{p} \sin \phi\right]$. (b) $\left(v_{\perp 1}, v_{\perp 2}\right)$-plane: Projection of a point $\left(v_{\perp 1}^{p}, v_{\perp 2}^{p}\right)$ (large red dot) representing an ion on a gyroorbit into the $\left(v_{\|}, v_{\perp 1}\right)$-plane. The $v_{\perp 1^{-}}$ coordinate is $v_{\perp}^{p} \cos \gamma^{p}$. The $u$-axis along $\mathbf{k}^{\delta}$ (blue) lies in the $\left(v_{\|}, v_{\perp 1}\right)$-plane and so is usually not identical to the $v_{\perp 1}$-axis.

where $v_{\|}^{p}$ and $v_{\perp}^{p}$ are constant and $\gamma^{p} \in\left[0,360^{\circ}\right]$ with respect to the $v_{\perp 1}$-axis. The gyroorbit then intersects the $\left(v_{\|}, v_{\perp 1}\right)$-plane at $\left(v_{\|}^{p}, v_{\perp}^{p}\right)$ for $\gamma^{p}=0^{\circ}$ and at its mirror image $\left(v_{\|}^{p},-v_{\perp}^{p}\right)$ for $\gamma^{p}=180^{\circ}$. For arbitrary gyroangles the projection into the $\left(v_{\|}, v_{\perp 1}\right)$ plane is $\left(v_{\|}^{p}, v_{\perp}^{p} \cos \gamma^{p}\right)$. It is then a matter of plane geometry to find the projected velocity $u^{p}$ as a function of the gyroangle $\gamma^{p}$ (see Fig. 2):

$$
u^{p}=v_{\|}^{p} \cos \phi+v_{\perp}^{p} \sin \phi \cos \gamma^{p} .
$$

A single point in $2 \mathrm{D}$ velocity space with coordinates $\left(v_{\|}^{p}, v_{\perp}^{p}\right)$ thus maps onto an interval of width $2 v_{\perp}^{p} \sin \phi$ centered on $v_{\|}^{p} \cos \phi$ in $u$-space as $\cos \gamma^{p}$ takes values from -1 to 1 . If the projection direction is perpendicular to the magnetic field $\left(\phi=90^{\circ}\right)$, the interval in $u$ is at its widest, going from $u^{p}=-v_{\perp}^{p}$ to $u^{p}=+v_{\perp}^{p}$, and is symmetric about $u=0$. In the other extreme, if the projection direction is parallel to the magnetic field $\left(\phi=0^{\circ}\right)$, the width of the interval goes to zero. In this case the point in $\left(v_{\|}, v_{\perp}\right)$-space will map onto a single point in $u$-space: $u^{p}=v_{\|}^{p}$. These intervals in $u$ determine in which frequency channels an ion with given $\left(v_{\|}^{p}, v_{\perp}^{p}\right)$ will elicit a response at given $\phi$.

Figure 3(a) shows three Maxwellian bumps as simple test velocity distribution functions in $\left(v_{\|}, v_{\perp 1}, v_{\perp 2}\right)$-space representing distinct populations of fast ions: trapped particles $(\mathrm{T})$, passing particles $(\mathrm{P})$ and particles near the trapped-passing-boundary (TP). These populations should be taken as illustrations only. The bulk part of a typical ITER plasma is modelled as isotropic deuterium Maxwellian distribution function with an ion temperature $T=20 \mathrm{keV}$ and an ion density $n=10^{20} \mathrm{~m}^{-3}$. Figures $3(\mathrm{~b})-(\mathrm{d})$ 
illustrate the projections of these velocity distribution functions onto $\mathbf{k}^{\delta}$. The projection directions, represented by the vector arrows and thin straight lines as in Fig. 1(a), are typical for the proposed ITER CTS system. The projections $g$ of the test functions onto these two projection directions are plotted in Figs. 3(b)-(d). For $\phi=100^{\circ}$ the intervals are much broader than for $\phi=10^{\circ}$ as their widths are $2 v_{\perp} \sin \phi$. Since the integral of $g$ always gives the fast-ion particle density (Eq. (3)) and is therefore the same for any $\phi$, the curves are flatter for $\phi=100^{\circ}$ than those for $\phi=10^{\circ}$. For $\phi=10^{\circ}$ the curves are more lopsided about $u=0$ than those for $\phi=100^{\circ}$ because the centre of each interval is $v_{\|} \cos \phi$.

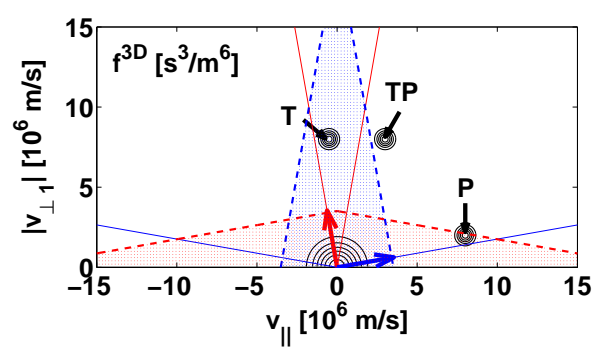

(a) Three bumps: T, P, TP.

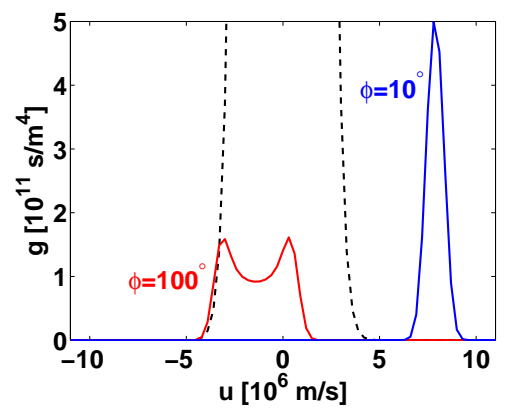

(c) Passing (P).

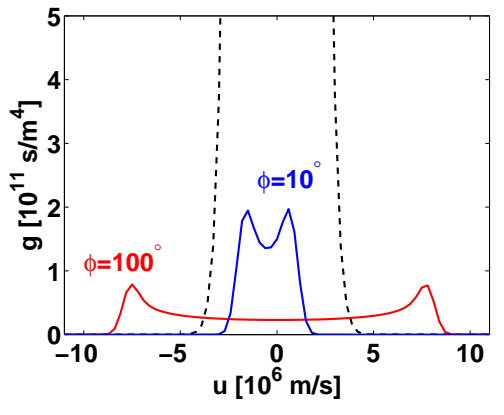

(b) Trapped (T).

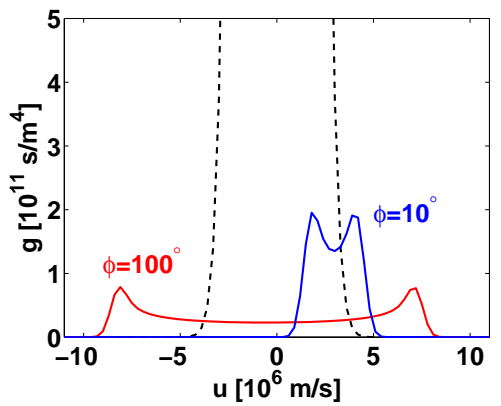

(d) TP-boundary (TP).

Figure 3. (a) Populations of trapped particles (T), passing particles (P), and particles near the trapped-passing-boundary (TP) are shown in a $2 \mathrm{D}$ slice of a 3D coordinate system. The concentric circles about the origin represent the bulk ion distribution. The projection directions used for (b)-(d) are shown as vector arrows and thin straight lines for $\phi=10^{\circ}$ (blue) and $\phi=100^{\circ}$ (red). These directions are typical for the forward scattering and backscattering systems, respectively. The dashed lines enclose triangular regions in which the much more numerous bulk ions hamper the detection of fast ions for each $\phi$. (b) Projections $g$ for the bump of trapped particles (T). (c) Projections $g$ for the bump of passing particles (P). (d) Projections $g$ for the bump of particles near the trapped-passing-boundary (TP). The dashed lines in (b)-(d) are the projections of the bulk plasma distribution function.

The projection of the isotropic Maxwellian bulk-ion distribution function is a Maxwellian (dashed lines in Figs. 3(b) to (d)) described by the bulk-ion temperature and particle density. As the bulk-ion particle density is much larger than that of fast ions, the scattered radiation related to bulk ions will obscure that of fast ions for values 
of

$$
|u|=\left|v_{\|} \cos \phi+v_{\perp} \sin \phi \cos \gamma\right|<A \times v_{t h},
$$

where the factor $A$ reflects a velocity level one chooses below which little fast ion information can be extracted (usually $A \sim 2$ ). The bulk ions thus create an obscure region - a triangle in $\left(v_{\|}, v_{\perp}\right)$-space or a bicone in $\left(v_{\|}, v_{\perp 1}, v_{\perp 2}\right)$-space - in which the fast ions are very difficult to detect. The shape of such triangles depends on $\phi$ and is given by the three sides

$$
\begin{aligned}
& v_{\perp}=\frac{A \times v_{t h} \pm v_{\|} \cos \phi}{\sin \phi}, \\
& v_{\perp}=0 .
\end{aligned}
$$

Two such triangles are plotted for $\phi=10^{\circ}$ and for $\phi=100^{\circ}$ in Fig. 3(a) as dashed lines enclosing shaded regions. The bump of trapped particles $(\mathrm{T})$ can be well detected with the backscattering system $\left(\phi \approx 100^{\circ}\right)$, but the bulk ions hamper detection with the forward scattering system $\left(\phi \approx 10^{\circ}\right)$. Co- and counter-passing particles $(\mathrm{P})$ with pitch near \pm 1 , on the other hand, are well detectable with the forward scattering system, but the bulk ions hamper detection with the backscattering system. Particles assumed to be near the trapped-passing-boundary (TP) in our illustration are well detectable with either system.

\section{Weight functions}

In Sec. 3 we discussed the question: Given an ion in 2D velocity space, where is it in $u$-space or in which frequency channels of the CTS receiver will it elicit a response? In this section we ask the reciprocal question: Given a fast-ion phase space density at a particular $u$ - or a detected spectral power density in a particular frequency channel where could the ions that caused the collective scattering be in $2 \mathrm{D}$ velocity space? The answer is expressed in terms of plasma independent weight functions which are positive in the velocity space interrogation regions and zero in the velocity space regions where the ion cannot be for given $u$ and $\phi$. Weight functions $w_{v}$ in $\left(v_{\|}, v_{\perp}\right)$-space are defined such that

$$
g(u, \phi)=\int_{-\infty}^{\infty} \int_{0}^{\infty} w_{v}\left(u, \phi, v_{\|}, v_{\perp}\right) f_{v}^{2 D}\left(v_{\|}, v_{\perp}\right) d v_{\perp} d v_{\|}
$$

or correspondingly $w_{E p}$ in $(E, p)$-space

$$
g(u, \phi)=\int_{-1}^{1} \int_{0}^{\infty} w_{E p}(u, \phi, E, p) f_{E p}^{2 D}(E, p) d E d p .
$$

The product of a plasma independent weight function and a fast-ion 2D distribution function of a particular plasma (the integrand in Eq. 8 or Eq. 9) represents the relative contributions of regions in 2D velocity space to $g$ for given $u$ and $\phi$.

To find analytic expressions for weight functions in $\left(v_{\|}, v_{\perp}\right)$-space, we compare Eq. 1 with Eq. 8. The $\left(v_{\|}, v_{\perp 1}, v_{\perp 2}\right)$-coordinate system is chosen such that the $v_{\|}$-axis 
is aligned with $\mathbf{B}$ and the $v_{\perp 1}$-axis lies in the plane spanned by $\mathbf{k}^{\delta}$ and $\mathbf{B}$ (Fig. 2). The $k_{\perp 2}$ component of $\mathbf{k}^{\delta}$ is then zero and Eq. 1 becomes

$$
\begin{aligned}
g(u, \phi) & =\int_{-\infty}^{\infty} \int_{-\infty}^{\infty} \int_{-\infty}^{\infty} f^{3 D}\left(v_{\|}, v_{\perp 1}, v_{\perp 2}\right) \delta\left(\frac{\mathbf{v} \cdot \mathbf{k}^{\delta}}{k^{\delta}}-u\right) d v_{\|} d v_{\perp 1} d v_{\perp 2} \\
& =\int_{-\infty}^{\infty} \int_{-\infty}^{\infty} \int_{-\infty}^{\infty} f^{3 D}\left(v_{\|}, v_{\perp 1}, v_{\perp 2}\right) \delta\left(v_{\|} \cos \phi+v_{\perp 1} \sin \phi-u\right) d v_{\|} d v_{\perp 1} d v_{\perp 2} \\
& =\int_{-\infty}^{\infty} \int_{0}^{\infty} f_{v}^{2 D}\left(v_{\|}, v_{\perp}\right) \frac{1}{2 \pi} \int_{0}^{2 \pi} \delta\left(v_{\|} \cos \phi+v_{\perp} \sin \phi \cos \gamma-u\right) d \gamma d v_{\perp} d v_{\|} .
\end{aligned}
$$

The expression for the weight function is by comparison with Eq. 8

$$
w_{v}^{a}\left(u, \phi, v_{\|}, v_{\perp}\right)=\frac{1}{2 \pi} \int_{0}^{2 \pi} \delta\left(v_{\|} \cos \phi+v_{\perp} \sin \phi \cos \gamma-u\right) d \gamma
$$

The superscript $a$ denotes that $w_{v}^{a}$ is an analytic expression. The weight function is positive at those locations in $\left(v_{\|}, v_{\perp}\right)$-space where a gyroangle $\gamma$ exists that satisfies Eq. 4 for the given $u$ and $\phi$. The argument of the Dirac delta function is then zero. If no such gyroangle exists for given $\left(u, \phi, v_{\|}, v_{\perp}\right)$, the delta function argument is never zero, and the weight function is then zero at $\left(v_{\|}, v_{\perp}\right)$ for the given $u$ and $\phi$. If a delta function argument has roots, the delta function can be rewritten using

$$
\delta(h(x))=\sum_{k} \frac{\delta\left(x-x_{k}\right)}{\left|h^{\prime}\left(x_{k}\right)\right|},
$$

where $x_{k}$ are the roots of $h$, and the prime denotes a derivative. If roots $\gamma_{k}$ of the delta function argument in Eq. 10 exist, they are given by

$$
\cos \gamma_{k}=\frac{u-v_{\|} \cos \phi}{v_{\perp} \sin \phi}
$$

and so the weight function can be written

$$
\begin{aligned}
& w_{v}^{a}\left(u, \phi, v_{\|}, v_{\perp}\right)=\frac{1}{2 \pi} \int_{0}^{2 \pi} \sum_{k} \frac{\delta\left(\gamma-\gamma_{k}\right)}{v_{\perp} \sin \phi \sin \gamma_{k}} d \gamma \\
= & \frac{1}{2 \pi v_{\perp} \sin \phi \sqrt{1-\left(\frac{u-v_{\|} \cos \phi}{v_{\perp} \sin \phi}\right)^{2}}} \sum_{k} \int_{0}^{2 \pi} \delta\left(\gamma-\gamma_{k}\right) d \gamma .
\end{aligned}
$$

There are then always two roots in the interval $[0,2 \pi]$ so that the weight function becomes

$$
w_{v}^{a}\left(u, \phi, v_{\|}, v_{\perp}\right)=\frac{1}{\pi v_{\perp} \sin \phi \sqrt{1-\left(\frac{u-v_{\|} \cos \phi}{v_{\perp} \sin \phi}\right)^{2}}} .
$$

Equation 15 is valid in the velocity space regions where the delta function argument in Eq. 10 has roots. These are the velocity space interrogation regions. Otherwise $w_{v}^{a}\left(u, \phi, v_{\|}, v_{\perp}\right)=0$, describing velocity space regions in which ions cannot be observed at given $u$ and $\phi$.

An alternative way to arrive at this result is by noting the geometrical relationship between $u$ and $\gamma$ illustrated in Fig. 2. Due to the assumed rotational invariance of $f^{3 D}$, every gyroangle of the ion is equally likely. We may consider the gyroangle $\gamma$ a random variable with the probability density function $p_{\gamma}(\gamma)=1 /(2 \pi)$. In this picture $u$ is also 
a random variable, and we get the probability density function of $u$, given $\phi, v_{\|}$and $v_{\perp}$, by the transformation

$$
p_{u}\left(u \mid \phi, v_{\|}, v_{\perp}\right)=p_{\gamma}(\gamma)\left|\frac{d \gamma}{d u}\right|,
$$

where the derivative is found from Eq. 4. The right hand side turns out identical to that of Eq. 15. The weight function is thus the probability density function of the random variable $u$, given $\phi, v_{\|}$and $v_{\perp}$.

The weight function $w_{v}^{a}\left(u, \phi, v_{\|}, v_{\perp}\right)$ is singular near its limits in $u$ for which the values are positive. However, the integral $\int w_{v}^{a}\left(u, \phi, v_{\|}, v_{\perp}\right) d u$ can easily be shown to be finite. It is unity as for every probability density function. We will exploit this fact to construct approximate weight functions $w_{v}^{b}$ by binning. The weight functions and their finite integrals explain the double-peak structure of the curves in Fig. 3.

It is useful to find these expressions in $(E, p)$-coordinates. Equation 4 expressed in $(E, p)$ is

$$
u=\left(p \cos \phi+\sqrt{1-p^{2}} \sin \phi \cos \gamma\right) \sqrt{2 E / m},
$$

and Eq. 15 expressed in $(E, p)$ is

$$
w_{E p}^{a}(u, \phi, E, p)=\frac{1}{\pi \sqrt{2 E / m\left(1-p^{2}\right)} \sin \phi \sqrt{1-\left(\frac{u / \sqrt{2 E / m}-p \cos \phi}{\sqrt{1-p^{2}} \sin \phi}\right)^{2}}}
$$

This result is consistent with the proportionality relation in the appendix of [36]. Here we give a complete analytic expression.

Figure 4 shows weight functions for several projection angles $\phi$ and a given velocity $u=6 \times 10^{6} \mathrm{~m} / \mathrm{s}$ as examples. The upper row shows weight functions in $\left(v_{\|}, v_{\perp}\right)$ coordinates, and the lower row corresponding weight functions in $(E, p)$-coordinates. The weight functions have positive values in the coloured regions which we call interrogation regions. Ions within these regions are observable for the given $u$ and $\phi$. On the other hand, ions in white regions, where the weight functions are zero, are unobservable. Explicit expressions for the limiting lines separating the velocity space interrogation regions from the unobservable regions in $\left(v_{\|}, v_{\perp}\right)$-space are found by solving Eq. 4 for $v_{\perp}$.

$$
v_{\perp}=\frac{u-v_{\|} \cos \phi}{\sin \phi \cos \gamma} .
$$

For each gyroangle $\gamma$, there is a line of constant slope in $\left(v_{\|}, v_{\perp}\right)$-space along which ions with that $\gamma$ project onto a given $u$ for a given $\phi$. Such lines are shown in Fig. 5. Two limiting lines with $\gamma=0^{\circ}$ or $\gamma=180^{\circ}$ are

$$
v_{\perp}= \pm \frac{u-v_{\|} \cos \phi}{\sin \phi}
$$

These two lines intersect the $v_{\|}$-axis at $v_{\|}=u / \cos \phi$ and have the slopes $\pm v_{\|} / \tan \phi$. Their closest distance to the origin is $u$. Points below these lines cannot map onto $u$. The weight functions are largest just above these lines at which the gyroangle is $\gamma=0^{\circ}$ or $\gamma=180^{\circ}$. These large values near the limits are expected [40]. 


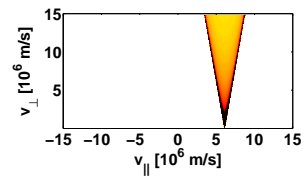

(a) $\phi=10^{\circ}$

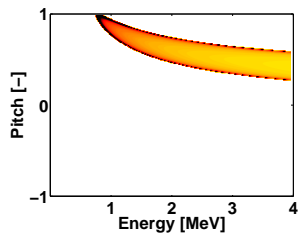

(e) $\phi=10^{\circ}$

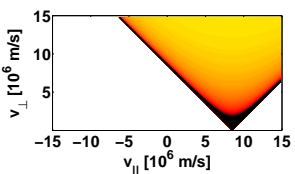

(b) $\phi=45^{\circ}$

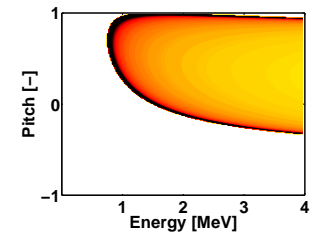

(f) $\phi=45^{\circ}$

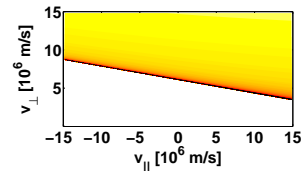

(c) $\phi=80^{\circ}$

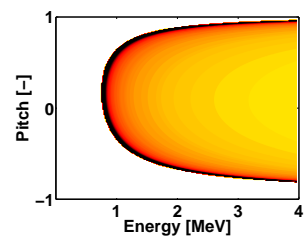

(g) $\phi=80^{\circ}$

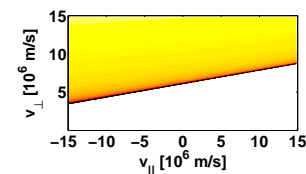

(d) $\phi=100^{\circ}$

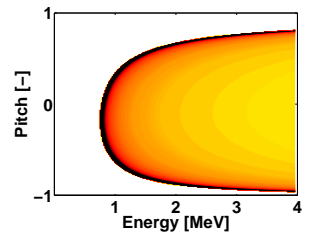

(h) $\phi=100^{\circ}$

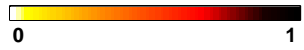

Figure 4. Weight functions in $\left(v_{\|}, v_{\perp}\right)$-coordinates $((\mathrm{a})-(\mathrm{d}))$ for $u=6 \times 10^{6} \mathrm{~m} / \mathrm{s}$ and various projection angles $\phi$. The corresponding weight functions in $(E, p)$-coordinates are shown in (e)-(f). The angles $\phi=10^{\circ}$ and $\phi=100^{\circ}$ are typical for the forwardand backscattering ITER CTS systems, respectively, while the angles $45^{\circ}$ and $80^{\circ}$ are shown for illustrative purpose. The coloured regions are interrogation regions while the white regions are unobservable for the given $\phi$ and $u$.

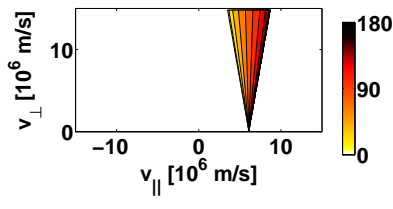

(a) $\phi=10^{\circ}$

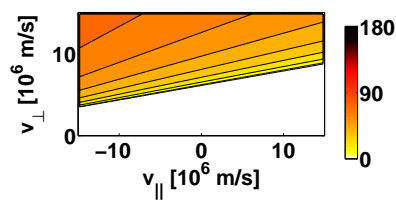

(b) $\phi=100^{\circ}$

Figure 5. Contours of the gyroangle within the interrogation regions shown in Fig. 4(a) and (d), respectively. The colours show the necessary gyroangle $\gamma \in\left[0^{\circ}, 180^{\circ}\right]$ to map onto $u=6 \times 10^{6} \mathrm{~m} / \mathrm{s}$ for the given $\phi$ in $\left(v_{\|}, v_{\perp}\right)$-space. For $\gamma \in\left[180^{\circ}, 360^{\circ}\right]$ similar maps can be found as $\cos \left(360^{\circ}-\gamma\right)=\cos \gamma$.

Weight functions $w_{v}$ for any $\phi$ (except for $\phi=0^{\circ}, 90^{\circ}, 180^{\circ}, 270^{\circ}$ ) and $u$ are qualitatively similar: they are triangles in $\left(v_{\|}, v_{\perp}\right)$-space (Figs. $\left.4(\mathrm{a})-(\mathrm{d})\right)$. If the $v_{\|^{-}}$ intercept $u / \cos \phi$ of the triangle is larger than any ion velocity (if $\phi \sim 90^{\circ}$ ), then only one of the limiting lines is relevant to the experiment such as for the proposed ITER CTS fast-ion system for $\phi \approx 100^{\circ}$ (Fig. 4(d)). The interrogation region then covers a fairly large region of the velocity distribution function in velocity space. To the contrary, the weight function picks out a narrow triangle in velocity space for the forward scattering system with $\phi \approx 10^{\circ}$ (Fig. 4(a)). The projection angles $\phi=45^{\circ}$ and $\phi=80^{\circ}$ are shown for illustrative purpose (Figs. 4(b)-(c)). For $\phi=45^{\circ}$ the triangle is wider than for $\phi=10^{\circ}$ and intercepts the $v_{\|}$-axis at larger values. For $\phi=80^{\circ}$ the triangle is yet wider and the $v_{\|}$-intercept yet larger, and the triangle is a mirror image of that for $\phi=100^{\circ}$. The seemingly different shapes of the four corresponding weight functions $w_{E p}$ in $(E, p)$ coordinates can likewise be explained (Figs. 4(e)-(h)). 
The analytic weight functions are singular on the limiting lines given by Eq. 20 and so are inconvenient for numerical work. However, Eq. 15 can be integrated over $u$, and the integral can be shown to be unity. This is also expected since the weight function is a probability density function. We can therefore find corresponding binning weight functions $w_{v}^{b}\left(u, \phi, v_{\|}, v_{\perp}\right)$ and $w_{E p}^{b}(u, \phi, E, p)$ by binning $u$ into intervals for $\gamma=[0,2 \pi]$ using Eq. 4. To do this we find contributions of Maxwellian bumps distributed in $\left(v_{\|}, v_{\perp}\right)$-space to a given $g(u)$ at the projected velocity $u$. Three such bumps were illustrated in Fig. 3. The weight functions are then normalized to satisfy Eq. 8. If the characteristic width of the Maxwellians and the bin width go to zero, the analytic weight functions are recovered with very large values near the limiting lines. We use a characteristic width corresponding approximately to the typical resolution of numerical simulations.

\section{Velocity space interrogation regions of the proposed forward- and backscattering ITER CTS systems}

Figure 6 shows example interrogation regions of a single CTS spectrum for a central measurement volume of the proposed backscattering system in a standard ITER burning plasma. The colours show the integrand of Eq. 9 (the relative contributions to $g$ ) for $\phi=100^{\circ}$ for eight example $u$ values. The central alpha distribution function has been computed with TRANSP. The integrands in the interrogation region tend to be larger towards the small energy values because the alpha distribution function tends to be larger there. We have not converged the statistics of the alpha distribution function to be completely smooth to show the effect of small-scale features in the alpha velocity distribution. The CTS signal will then come mostly from those regions within the interrogation regions where the alphas are most numerous and the weight functions are largest. The regions without colours are unobservable with the CTS diagnostic at the given $u$ and $\phi$. The positive and negative velocities $u$ interrogate similar regions for the backscattering system, and the 1D projections $g$ will then be nearly symmetric. Figure 7 shows corresponding plots for the proposed forward scattering system. Copassing ions with positive pitch at several energies can be measured for positive $u$ and counter-passing ions with negative pitch for negative $u$. The interrogation regions of the forward scattering system cover a narrower region in $(E, p)$-space compared with those of the backscattering system.

While the weight functions are plasma independent, the integrand of Eq. 9 is also proportional to the particular fast-ion distribution function. A comparison of Fig. 8(a) with Fig. 7(a) and Fig. 8(b) with Fig. 6(a) illustrates that the region from which most of the CTS signal at the given $u$ and $\phi$ originates - the region which is effectively probed - can be very different for other fast ion distributions. The weight functions in each pair are identical, but it is multiplied with the alpha distribution in Figs. 6 and 7 and with the NBI distribution from Fig. 1 in Fig. 8. 

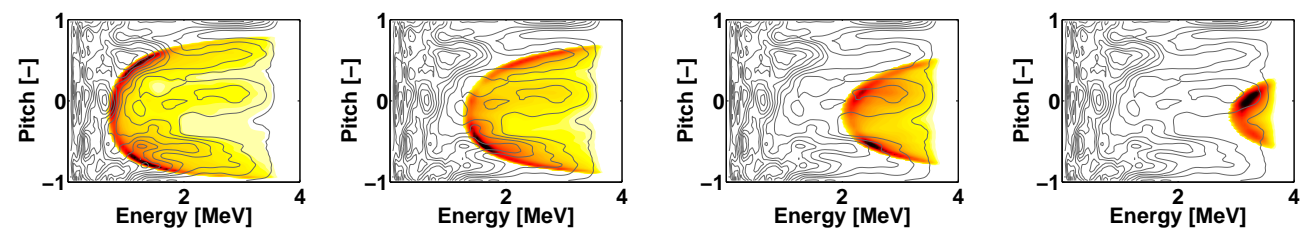

(a) $u=6 \times 10^{6} \mathrm{~m} / \mathrm{s}$

(b) $u=8 \times 10^{6} \mathrm{~m} / \mathrm{s}$

(c) $u=10 \times 10^{6} \mathrm{~m} / \mathrm{s}$

(d) $u=12 \times 10^{6} \mathrm{~m} / \mathrm{s}$
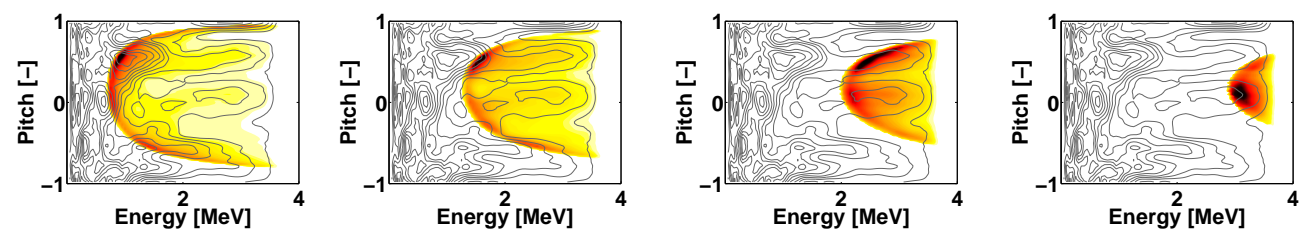

(e) $u=-6 \times 10^{6} \mathrm{~m} / \mathrm{s}$

(f) $u=-8 \times 10^{6} \mathrm{~m} / \mathrm{s}$

(g) $u=-10 \times$

(h) $u=-12 \times$ $10^{6} \mathrm{~m} / \mathrm{s}$

$10^{6} \mathrm{~m} / \mathrm{s}$

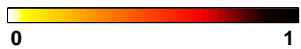

Figure 6. Relative contributions from the interrogation regions in $(E, p)$-space for $\phi=100^{\circ}$ and various velocities $u$. The coloured regions show the integrand of Eq. 9, i.e. the product of the weight functions and a central alpha distribution function $(r / a=0)$ computed with TRANSP. The integrands have been normalized. The alpha distribution function is illustrated as grey isocontours.
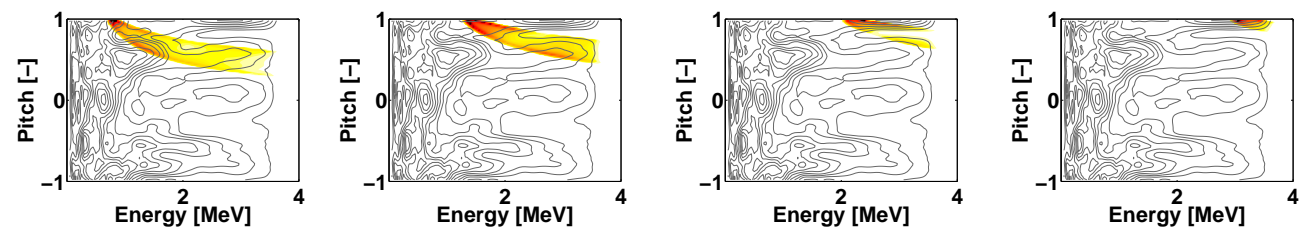

(a) $u=6 \times 10^{6} \mathrm{~m} / \mathrm{s}$

(b) $u=8 \times 10^{6} \mathrm{~m} / \mathrm{s}$

(c) $u=10 \times 10^{6} \mathrm{~m} / \mathrm{s}$

(d) $u=12 \times 10^{6} \mathrm{~m} / \mathrm{s}$
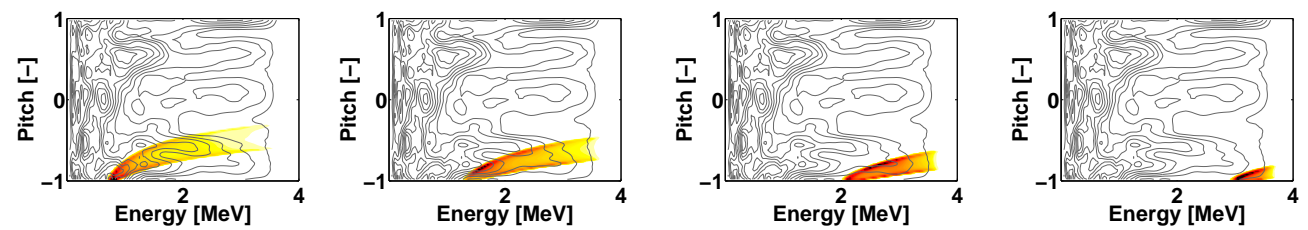

(e) $u=-6 \times 10^{6} \mathrm{~m} / \mathrm{s}$

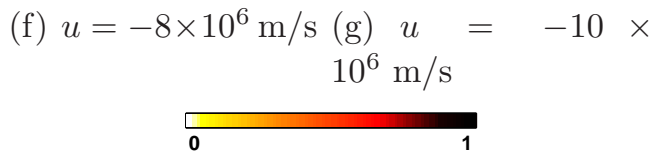

(h) $u=-12 \times$ $10^{6} \mathrm{~m} / \mathrm{s}$

Figure 7. Relative contributions from the interrogation regions in $(E, p)$-space for $\phi=10^{\circ}$ and various velocities $u$. The coloured regions show the integrand of Eq. 9, i.e. the product of the weight functions and a central alpha distribution function $(r / a=0)$ computed with TRANSP. The integrands have been normalized. The alpha distribution function is illustrated as grey isocontours. 


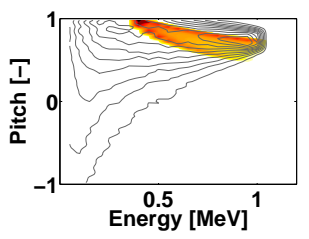

(a) $\phi=10^{\circ}$

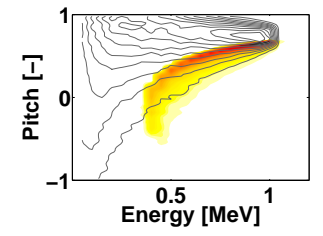

(b) $\phi=100^{\circ}$

0

Figure 8. Relative contributions from the interrogation regions in $(E, p)$-space for $\phi=10^{\circ}$ and $\phi=100^{\circ}$ with $u=6 \times 10^{6} \mathrm{~m} / \mathrm{s}$. The coloured regions show the integrand of Eq. 9, i.e. the product of the weight functions and the high field side NBI distribution function at $r / a=0.25$ computed with TRANSP (see Fig. 1(d)). The integrands have been normalized. The NBI distribution function is illustrated as grey isocontours.

\section{Reconstruction of the fast-ion velocity distribution function from two 1D projections}

Weight functions can be used to reconstruct the fast-ion velocity distribution function from two 1D distributions functions along different projection directions. This problem has no unique solution [41], but it is possible to find reasonable reconstructions. Here we present an iteration algorithm to find such reconstructions by exploiting the knowledge contained in weight functions. Figure 9 (a) shows three bumps in $(E, p)$-space as simple test model, and Fig. 9(d) shows the NBI ion distribution function from Fig. 1 as a second test model. The aim is to reconstruct these target 2D test functions, given only two 1D projections $g$ with different projection angles. In an actual experiment, two target 1D projections $g$ can be obtained from two simultaneous CTS measurements with different projection angles. Here we choose the angles to be $\phi=10^{\circ}$ and $\phi=100^{\circ}$ corresponding to the forward- and backscattering ITER CTS systems and use only information that would not be masked by the bulk ions in ITER and are hence outside the shaded triangles in Fig. 3, i.e. $|u|>3 \times 10^{6} \mathrm{~m} / \mathrm{s}$. After an initial guess, two projections $g$ are computed from the iterated $2 \mathrm{D}$ velocity distribution function. The next iteration is obtained by adding or subtracting small multiples of the weight functions for each $u$ and $\phi$ to the 2 D velocity distribution function, depending on whether the corresponding iterated $g$ is larger or smaller than the target $g$, respectively. The iterated solution depends on the initial guess for which we choose zeros everywhere. Reasonable reconstructions are then reliably obtained for a wide range of target functions compared with other initial

guesses. Fig. 9 demonstrates that large-scale features of the simple 2D test functions can reasonably be reconstructed, given only two 1D projections with $|u|>3 \times 10^{6} \mathrm{~m} / \mathrm{s}$ (Figs. 9(b) and (e)).

To demonstrate that measurements from other fast-ion diagnostics could be included in the algorithm, we use a third $g$ for a hypothetical third projection angle $\left(\phi=45^{\circ}\right)$ for the reconstruction (Figs. 9(c) and (f)). This CTS geometry is not feasible in ITER due to refraction. However, the hypothetical extra CTS measurement 
serves as example for the possible extension of the algorithm. The reconstruction of the more complicated NBI ion distribution function improves due to the extra information. The reconstruction algorithm and its extension will be used in future experiments at ASDEX Upgrade where a second CTS receiver is currently being installed and FIDA is available [42].

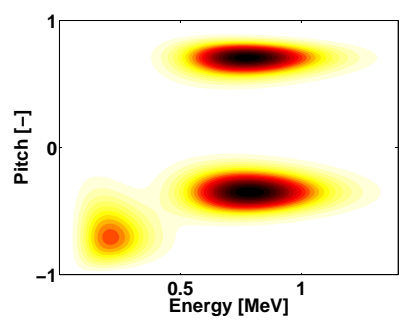

(a) Target function.

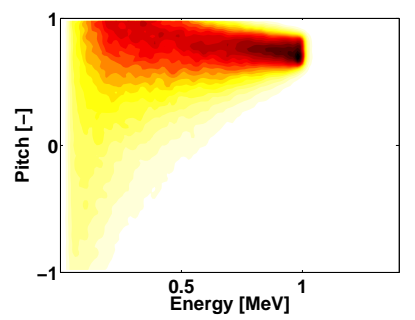

(d) Target function.

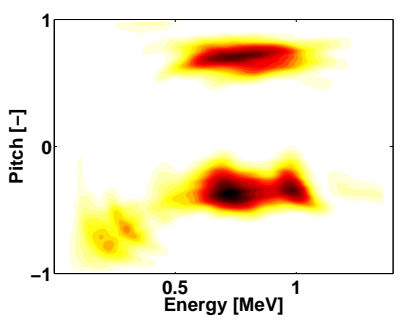

(b) Two CTS measurements.

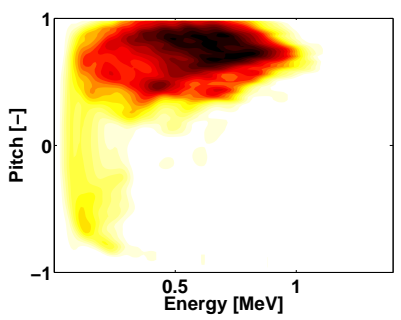

(e) Two CTS measurements.

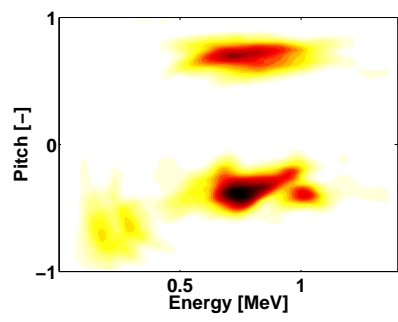

(c) Three CTS measurements.

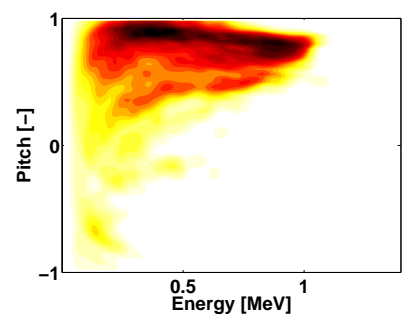

(f) Three CTS measurements.

Figure 9. Reconstruction of a target function ((a) and (d)) given only the information obtained from two 1D projections $g((\mathrm{~b})$ and (e)) or three 1D projections $g((\mathrm{c})$ and (f)) with $|u|>3 \times 10^{6} \mathrm{~m} / \mathrm{s}$. The projection angles of the two $1 \mathrm{D}$ projections were $\phi=10^{\circ}$ and $\phi=100^{\circ}$ in (b) and (c). In (c) and (f) additionally a projection with $\phi=45^{\circ}$.

\section{Conclusions}

Here we show the velocity space interrogation regions of the proposed ITER CTS system. The backscattering system with receivers on the low field side is an enabled ITER diagnostic whereas the forward scattering system with receivers on the high field side is not. The backscattering system is sensitive to fast ions with pitch $|p|<0.5-0.9$, depending on the ion energy and the frequency shift of the scattered radiation. Its viewing geometry makes it easy to retrofit it with a CTS-based fuel ion ratio diagnostic [43-46]. On the other hand, the forward scattering system would be sensitive to co- and counter-passing fast ions at various energies with pitch $|p|>0.6-0.8$. Its interrogation regions in $2 \mathrm{D}$ velocity space are narrow compared with those for the backscattering system which cover broad ranges in $(E, p)$-space. The relative contributions of different regions in $2 \mathrm{D}$ velocity space to the CTS signal have been computed using weight functions and TRANSP simulations of the alpha and NBI ion distributions in ITER, 
showing the regions which are effectively probed. The interrogation regions are described by weight functions. We here derive analytic expressions for the weight functions which should also be useful for FIDA since CTS weight functions are identical to the geometric (Doppler shift) part of FIDA weight functions [35-38]. Moreover, it is shown that weight functions can efficiently be used as base functions in reconstructions of $2 \mathrm{D}$ fastion distribution functions, given two simultaneous CTS measurements with different scattering geometries. The quality of such reconstructions can be improved by including fast-ion measurements from other diagnostics in the iteration algorithm. Thus, we have shown the benfits of the back- and forward scattering ITER CTS systems and their combination. Much added information could be gained from a forward scattering system, allowing measurements of co- and counter-passing ions with high velocity space resolution, revealing anisotropy in the fast-ion populations and even allowing reconstruction of 2D fast-ion distribution functions.

\section{Acknowledgments}

This work, supported by the European Communities under the contract of Association between Euratom and Ris $\varnothing$ DTU was partly carried out within the framework of the European Fusion Development Agreement. The views and opinions expressed herein do not necessarily reflect those of the European Commission. We acknowledge the discussions on this topic within the Integrated Tokamak Modelling Activities (ITPA) group for Energetic Particles which initiated this study.

\section{References}

[1] Chen L 1994 Phys. Plasma 1 1519-1522

[2] Briguglio S et al 2007 Phys. Plasma 14 055904-055904-10

[3] Heidbrink W W and Sadler G J 1994 Nucl. Fusion 34535

[4] Zweben S J et al 2000 Nucl. Fusion 4091 - 149

[5] Heidbrink W W 2002 Phys. Plasma 92113 - 2119

[6] Pinches S D et al 2004 Plasma Phys. Control. Fusion 46 B187-B200

[7] Gorelenkov N N, Berk H L and Budny R V 2005 Nucl. Fusion 45 226-237

[8] Campbell D J et al 1988 Phys. Rev. Lett. 60 2148-2151

[9] Marcus F B et al 1994 Nucl. Fusion 34(5) 687-701

[10] Nielsen S K et al 2010 Plasma Phys. Control. Fusion 52092001

[11] Nielsen S K et al 2011 Nucl. Fusion 51063014

[12] Chang Z et al 1996 Phys. Rev. Lett. 761071

[13] Nazikian R et al 1996 Phys. Plasma 3(2) 593-605

[14] García-Muñoz M et al 2007 Nuclear Fusion 47 L10-L15

[15] Günter S et al 2007 Nucl. Fusion 47 920-928

[16] Hauff T, Pueschel M J, Dannert T and Jenko F 2009 Phys. Rev. Lett. 102075004

[17] Heidbrink W W et al 2009 Phys. Rev. Lett. 103175001

[18] ITER Physics Expert Group on Energetic Particles, Heating and Current Drive 1999 Nucl. Fusion 39 2471-2495

[19] Fasoli A et al Progress in the ITER Physics Basis Chapter 5: Physics of energetic ions 2007 Nucl. Fusion 47 S264 
[20] Bindslev H et al 1999 Phys. Rev. Lett. 83(16) 3206-3209

[21] Bindslev H et al 2006 Phys. Rev. Lett. 97205005

[22] Nielsen S K et al 2008 Phys. Rev. E 77016407

[23] Moseev D et al 2011 Plasma Phys. Control. Fusion Comparison of measured and simulated fast ion velocity distributions in the TEXTOR tokamak, submitted

[24] Meo F et al 2008 Rev. Sci. Instrum. 79 10E501

[25] Salewski M et al 2010 Nucl. Fusion 50035012

[26] Meo F et al 2010 J. Phys.: Conf. Series 227012010

[27] Meo F et al 2004 Rev. Sci. Instrum. 75 3585-3588

[28] Korsholm S B et al 2008 Burning Plasma Diagnostics (AIP conference proceedings vol 988) pp 118-122

[29] Salewski M et al 2008 Rev. Sci. Instrum. 79(10) 10E729

[30] Salewski M et al 2009 Plasma Phys. Control. Fusion 51035006

[31] Salewski M et al 2009 Nucl. Fusion 49025006

[32] Leipold F et al 2009 Rev. Sci. Instrum. 80093501

[33] Donne A J H et al Progress in the ITER Physics Basis Chapter 7: Diagnostics 2007 Nucl. Fusion $47 \mathrm{~S} 337$

[34] Costley A et al in Fusion Energy 2008 (Proc. 22nd Int. Conf. Geneva, 2008) (Vienna: IAEA) CDROM file IT/1-5 and http://www-naweb.iaea.org/napc/physics/FEC/FEC2008/html/index.htm

[35] Luo Y et al 2007 Rev. Sci. Instrum. 78033505

[36] Heidbrink W W et al 2007 Plasma Phys. Control. Fusion 49 1457-1475

[37] van Zeeland M A, Heidbrink W W and Yu J H 2009 Plasma Phys. Control. Fusion 51055001

[38] Heidbrink W W 2010 Rev. Sci. Instrum. 81 10D727

[39] Pankin A, McCune D, Andre R and Kritz A 2004 Comp. Phys. Communications 159 157-184

[40] Heidbrink W W 1984 Nucl. Fusion 24636

[41] Egedal J and Bindslev H 2004 Phys. Plasma 11(5) 2191-2198

[42] Geiger B et al 2011 Plasma Phys. Control. Fusion 53065010

[43] Korsholm S B et al 2010 Rev. Sci. Instrum. 81(10) 10D323

[44] Stejner M et al 2010 Rev. Sci. Instrum. 81(10) 10D515

[45] Korsholm S B et al 2011 Phys. Rev. Lett. 106165004

[46] Korsholm S B et al 2010 Nucl. Instrum. Methods Phys. Res. A 623677 